Pub. Mat. UAB

$N^{\circ} 21$ 0ct. 1980

Actes VII JMHL

ALGUNAS CARACTERIZACIONES DE LA CUADRICA COMPLEJA POR EL ESPECTRO DE SU LAPLACIANO

Manuel Barros

Dpto. de Geometría y Topología

Universidad de Granada

Introducción.

Sea $\left(C p^{n+q}, J_{0}, g_{0}\right)$ el espacio proyectivo complejo de dimensión compleja $n+q$ con la estructura compleja natural Jo y la métrica de Fubini-Study go de curvatura seccional holomorfa constante 1 . Se define $Q_{n}=\left(\left\{\left(z_{0}, \ldots, z_{n+q}\right)_{E} C P^{n+q} / z_{0}^{2}+\ldots\right.\right.$ $\left.\ldots z_{n+q}^{2}=0\right\}$ jonde $\left\{z_{0}, \ldots, z_{n+q}\right\}$ es un sistema de coordenadas homogéneas sobre $C P^{n+q}$. Es bien conocido que con respecto a la métrica inducida $g_{0}$, $Q_{n}$ es una variedad Kaehleriana Einstein y usualmente es llamada la cuâdrica compleja de dimensión compleja $n$.

Se considera el siguiente problema:"Sea $(M, J, g)$ una variedad Kaehleriana compacta de dimensión compleja $n$, supongamos que para algún p fijo $(0 \leqslant p \leqslant n)$ se verifica que $\operatorname{Spec}^{P}(M, g)=\operatorname{Spec}^{P}\left(Q_{n}, g_{\Omega}\right)$, entonces $i E s(M, J, g)$ holomórficamente isométríca a $\left(Q_{n}, J_{0}, g_{0}\right) ? "$.

En esta nota se dan algunas respuestas parciales obtenidas por el. autor $y$ B.Y.Chen al problema anterior, [1],[2],[3] y [4],El problema general sigue abierto.Tambien queremos señalar que es la primera vez que un espacio que no tiene curvatura constante (curvatura seccional holomorfa constante) se caracteriza por el espectro de su Laplaciano. 


\section{Resultados extrínsecos.}

En esta sección se consideran fundamentalmente subvariedades complejas y compactas del espacio proyectivo complejo.

Teorena I. - Sea $(M, J, g)$ una subvariedad compleja y compacta de dimensión compleja n en $\left(C \mathrm{P}^{\mathrm{n}+\mathrm{q}}, J_{0}, \mathrm{~g}_{\mathrm{O}}\right)$. Supongamos que $\mathrm{Spec}^{\mathrm{p}}(\mathrm{M}, \mathrm{g})=$ $=\operatorname{Spec}^{p}\left(Q_{n}, g_{0}\right)$, donde $g$ es la métrica inducida sobre $M$. Entonces, $(M, J, g)$ es holomórficamente isométrica a $\left(Q_{n}, J_{0}, g_{0}\right)$ en los siguientes casos:

(1) Si. $p=1$ y $8 \leqslant n \leqslant 15,[3]$

(2) Si $p=1,3 \leqslant n y(M, J, g)$ es cohomológicamente Einstein, [3]

(5) Si $\mathrm{p}=2$ y $\mathrm{n} \geqslant 5,[2]$

(4) Si $p=n$ y $n \geqslant 3,[3]$

(5) Para todo p y para todo $n$, si M está embebida en $C p^{n+q}$, [2] Un resultado intrinseco.

E] siguiente resultado ha sido anunciado en [2], y nosotros daremos un esquena de una demostración del mismo.

Teorema II.- Sea $(M, J, g)$ una superficie Kaehleriana y compacta con $\operatorname{Spec}^{\circ}(M, g)=\operatorname{Spec}^{\circ}\left(Q_{2}, g_{0}\right)$. Si $\times(M) \geqslant 4$, entonces $(M, J ; g)$ es holomórficamente jsométrica a $\left(Q_{2}, J_{0}, g_{0}\right)$. Donde $x(M)$ representa la caracteristica de Euler de $M$.

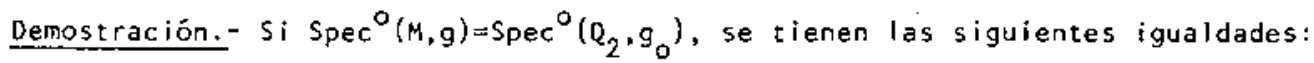

(1) $\operatorname{vol}(M, g)=\operatorname{vol}\left(Q_{2}, g_{0}\right) \quad$ (11) $\int_{M} 0: 1=\int_{Q_{2}} 0_{0}: 1$

(11) $\int_{M}\left\{2|R|^{2}-2|S|^{2}+5 \rho^{2}\right\} \div 1=\int_{Q_{2}}\left\{2\left|R_{O}\right|^{2}-2\left|S_{O}\right|^{2}+5 \rho_{O}^{2}\right\} \neq 1$

donde vol, $\rho, S$ y $R$ son respectivamente el volumen, la curvatura escalar, el tensor 
de Ricci y el tensor curvatura Riemanniano de $(M, g)$ y análogamente para $\left(Q_{2}, g_{o}\right)$. Además como $x(M) \geqslant 4$, se tiene que

(Iv) $\int_{M}\left\{|R|^{2}-4|S|^{2}+p^{2}\right\} \div 1 \geqslant \int_{Q_{2}}\left\{\left|R_{0}\right|^{2}-4|S|_{0}^{2}+0_{0}^{2}\right\}: 4$

Combinando $(I+I)$ y $(1 V)$, se tiene que $(M, J, g)$ es una superficie Kaehleriana y Einstein. Ademäs por (11), ella tiene curvatura escalar iqual a la de $Q_{2}$ y por tanto su tensor de Ricci es definido positivo.

Utilizando el tercer coeficiente $a_{3}$ del desarrollo asintótico de Minakshisundaran-pleijel-Gaffney para $\operatorname{Spec}^{\circ}(M, g)$ el cual ha sido calculado por Sakai,[7], no es dificil ver que $(M, q)$ es un espacio localmente simétrico. Ahora se utiliza un resultado bien conocido de $S$.Kobayashi,[6], y se tiene que $M$ debe ser simplemente, conexa.

Después de tado esto, $(M, J, q)$ es un espacio simétrico Hermítico el cual debe ser reducible pues en caso contrario sería el plano proyectivo complejo lo cual contradice por ejemplo (II). Asi se concluye que $(M, J, g)$ debe ser holomórficamente isométrica a $\left(Q_{2}, J_{0}, g_{0}\right)$.

\section{Referencias.}

[1]M.Barros, "Characterization of the complex sphere by its spectrum', Differential Geometry Seminar, Michigan State University. Sept. (1979).

[2]M.Barros and B.Y. Chen, "Complex quadrics and Spectral Geometry" Geometriae Dedicata (a aparecer).

[3]M.Barros,"Some extrinsic characterization of complex quadrics by its spectrum" (a aparecer).

[4]M. Barros,"Some characterizations of complex imnersions in the complex projective space by its spectrum", Conf. Univ. de Limoges. Mayo (1980). [5\}S.Helgason, "Differential Geometry,Lie groups and symmetric spaces" Academic press,N.Y., $\$ 978$. 
(6) S. Kobayashi,"On compact Kaehler manifolds with definite Ricci tensor" Ann. of Math. 74 (1961),570-574.

[7]T.Sakai,"On eigenvalues of Laplacian and curvature of Riemannian manifolds" Tohoku Math.J. 23 (1971), 589-603. 